\title{
Vigilância do desenvolvimento de crianças em creche: um estudo sobre o olhar materno
}

\author{
Surveillance of child development in a daycare center: a study on the maternal perspective \\ Vigilancia del desarrollo infantil en la guardería: un estudio sobre la mirada materna
}

\begin{abstract}
Altamira Pereira da Silva Reichert ${ }^{1}$ (1)
Tayná Lima dos Santos ${ }^{2}$ (1)

Daniele Beltrão Lucena de França ${ }^{1}$ (C)

Daniele de Souza Vieira ${ }^{1}$ (D)

Anniely Rodrigues Soares ${ }^{2}$ (D)
\end{abstract}

\section{Pós-graduação em Enfermagem da}

Universidade Federal da Paraíba. João Pessoa, Paraíba, Brasil.

2. Universidade Federal da Paraíba, Graduação em Enfermagem, João Pessoa, Paraíba, Brasil.
Autor correspondente:

Tayná Lima dos Santos

E-mail: taynalima95@hotmail.com

Recebido em 29/10/2020.

Aprovado em 17/03/2021.

\section{Resumo}

Objetivo: Compreender a percepção materna acerca da vigilância do desenvolvimento de crianças menores de três anos que frequentam creche. Método: Estudo qualitativo com nove mães de crianças menores de três anos, matriculadas em uma creche do município de João Pessoa-PB, vinculada a uma Unidade de Saúde da Família. Os dados foram coletados por meio de entrevistas semiestruturadas e submetidos à análise temática. Resultados: A vigilância do desenvolvimento de crianças que frequentam creche encontra-se fragilizada, visto que a maioria das mães não costumam levar a criança para a unidade de saúde, para o acompanhamento do crescimento e desenvolvimento, e a inserção dos profissionais de saúde na creche ainda é limitada. Entretanto, as mães reconhecem a creche como cenário ideal para a promoção do desenvolvimento infantil e a importância da atuação dos profissionais de saúde nesse cenário. Conclusão e implicações para prática: São necessárias ações conjuntas da creche com a Unidade de Saúde da Família para a atenção integral e vigilância do desenvolvimento infantil.

Palavras-chave: Saúde da Criança; Crescimento e Desenvolvimento; Estratégia Saúde da Família; Creches; Mães.

\section{Abstract}

Objective: To understand the maternal perception about the surveillance of the development of children under three years old who attend a daycare center. Method: A qualitative study with nine mothers of children under the age of three, enrolled in a daycare center in João Pessoa-PB, linked to a Family Health Unit. Data was collected through semi-structured interviews and subjected to thematic analysis. Results: The monitoring of the development of children attending a daycare center is weakened, since most mothers do not take the child to childcare in order to monitor growth and development, and the inclusion of health professionals in the daycare center is still limited. However, the mothers recognize the daycare center as an ideal setting for the promotion of child development and the importance of health professionals in this setting. Conclusion and implications for the practice: Joint actions of the daycare center with the Family Health Unit are necessary for comprehensive care and surveillance of child development.

Keywords: Child Health; Growth and Development; Family Health Strategy; Child Daycare Centers; Mothers.

\section{Resumen}

Objetivo: Comprender la percepción materna sobre la vigilancia del desarrollo de los niños menores de tres años que asisten a la guardería. Metodo: Estudio cualitativo con nueve madres de niños menores de tres años, inscriptos en una guardería de la ciudad de João Pessoa-PB, vinculada a una Unidad de Salud de la Familia. Los datos se recopilaron a través de entrevistas semiestructuradas y se sometieron a análisis temático. Resultados: La vigilancia del desarrollo de los niños que asisten a la guardería se debilita, ya que la mayoría de las madres no llevan al niño a la guardería, para monitorear el crecimiento y desarrollo, y la inclusión de profesionales de la salud en la guardería aún es limitada. Sin embargo, las madres reconocen la guardería como un escenario ideal para la promoción del desarrollo infantil y la importancia del desempeño de los profesionales de la salud en este ámbito. Conclusión e implicaciones para la práctica: Son necesarias acciones conjuntas de la guardería y la Unidad de Salud de la Familia para lograr la atención integral y vigilancia del desarrollo infantil.

Palabras clave: Salud del Niño; Crecimiento y Desarrollo; Estrategia de Salud Familiar; Jardines Infantiles; Madres. 


\section{INTRODUÇÃO}

Os avanços científicos e tecnológicos, as mudanças nos modelos assistenciais e a preocupação com a qualidade de vida e com os direitos humanos proporcionaram mudanças significativas na assistência à saúde da criança, com o intuito de evitar possíveis agravos e reduzir a mortalidade infantil. Assim, a atenção à saúde na infância vem alcançando espaços importantes nas políticas públicas, com propostas de ações em diferentes contextos da atenção, como o acompanhamento do desenvolvimento, principalmente na primeira infância, que corresponde ao período de zero a seis anos de idade. ${ }^{1}$

Nessa fase, há intensas modificações no desenvolvimento físico e neuropsicomotor da criança, exigindo cuidado contínuo e norteado pelo acompanhamento regular do crescimento e desenvolvimento, a fim de identificar previamente possíveis agravos a sua saúde. ${ }^{2}$ Assim, os primeiros anos são fundamentais para a Vigilância do Desenvolvimento Infantil (VDI).

Estima-se que, no mundo, 200 milhões de crianças com idade inferior aos cinco anos estão em risco de não alcançar o seu potencial de desenvolvimento. ${ }^{3}$ Dessa forma, salienta-se a importância das ações de VDI que buscam, por meio da avaliação profissional, identificar possíveis alterações no desenvolvimento, estimular e direcionar o cuidado adequado à criança. ${ }^{2}$ Para tanto, o acompanhamento infantil é fundamental, principalmente durante os três primeiros anos de vida, tendo em vista que representa a faixa etária de maior plasticidade cerebral e possibilidade de reversão de atrasos, em tempo oportuno. ${ }^{4}$

Nesse contexto, destacam-se os Centros de Referência em Educação Infantil (CREI), também denominados creches, locais onde há concentração de crianças em faixas etárias importantes para a realização da vigilância do crescimento e desenvolvimento, tornando-se ambientes fundamentais para o cuidado infantil. Esses espaços possibilitam a identificação de fatores de risco para atrasos no desenvolvimento, dentre eles, o nível socioeconômico, a moradia, escolaridade dos pais/cuidadores e a vulnerabilidade infantil. ${ }^{5}$

É evidente o crescimento expressivo na inserção de crianças nos centros de educação infantil, ${ }^{6}$ com aumento de $84,6 \%$ no número de matrículas em creches brasileiras nos últimos oito anos, ${ }^{7}$ acompanhando a tendência mundial que teve um crescimento de $64,0 \%$ de matrículas entre os anos de 1999 a $2012 .^{6}$

Contudo, para ser um ambiente favorável ao desenvolvimento das crianças, as creches devem ofertar serviços com qualidade capazes de promover a VDI. Conforme estudo, ${ }^{8}$ a qualidade do ambiente da creche, isto é, a estrutura física, os recursos humanos disponíveis e o cuidado dispensado à criança, somado a outros fatores extrínsecos, como maior escolaridade materna e maior tempo de permanência na creche, influenciam positivamente o desenvolvimento motor de crianças que frequentam esse ambiente educacional.

Frente à relevância dos CREI, a Lei das Diretrizes e Bases da Educação Nacional enfatiza o papel dos professores envolvidos com a educação infantil, primeira etapa da educação básica, para a promoção do desenvolvimento integral da criança de até cinco anos, em seus aspectos físico, psicológico, intelectual e social, complementando a ação da família e da comunidade. ${ }^{9}$ Assim, as creches ou entidades equivalentes tornam-se cenários privilegiados para a vigilância do desenvolvimento infantil.

Pensando nisso, os Ministérios da Saúde e da Educação implantaram o Programa Saúde na Escola (PSE), no ano de 2007, e um dos seus eixos diz respeito à avaliação clínica e psicossocial, na qual, dentre outras, estão contempladas a avaliação antropométrica, atualização do calendário vacinal, avaliação oftalmológica, auditiva, nutricional e da saúde bucal. ${ }^{10}$ Ressalta-se que essas ações também fazem parte da rotina da puericultura na atenção básica. Portanto, compreendendo que o PSE representa o elo entre a Unidade Básica de Saúde (UBS) e as escolas da educação básica, é fundamental que as ações de saúde propostas no programa também sejam realizadas nos $\mathrm{CREI}$.

As consultas de puericultura na atenção básica são realizadas por médicos e enfermeiros. ${ }^{11}$ A atuação destes profissionais frente às necessidades da criança, que está inserida na creche, tem grande valor para a longitudinalidade do cuidado dessa. Apesar disso, percebe-se que estes profissionais ainda possuem atuação limitada nos CREI, e esse pode ser um dos motivos para falhas na vigilância do crescimento e desenvolvimento de crianças na primeira infância, ${ }^{12}$ especialmente daquelas que frequentam creches.

Além disso, percebe-se na prática que as mães não costumam levar seus filhos que frequentam os CREI para a consulta de puericultura, corroborando estudo que destaca que o não comparecimento às consultas de puericultura na Estratégia Saúde da Família (ESF) é um problema entre as crianças matriculadas em creches, o que pode vir a comprometer a vigilância do desenvolvimento dessas. ${ }^{5}$

Ademais, destaca-se o papel das mães na promoção do desenvolvimento infantil saudável, tendo em vista que a interação da criança com essas molda as suas experiências. A criança relaciona-se com a família e com a comunidade, incluindo a creche. Assim, o cuidado responsivo no domicílio e na creche e as interações enriquecedoras e protetoras proporcionam um ambiente ideal para o seu desenvolvimento e protegem a criança dos efeitos negativos das adversidades. ${ }^{13}$

Familiares e cuidadores exercem papel fundamental para a VDI também na creche, desde que compreendam e apoiem, quando necessário, a atuação dos profissionais da creche e da saúde nesse cenário educacional. Portanto, a participação da família se torna indispensável para um bom desenvolvimento infantil. ${ }^{14}$

Diante do exposto, o presente estudo justifica-se pela importância em investigar a existência do acompanhamento do desenvolvimento infantil por profissionais da saúde em creches e seu significado para as mães, tendo em vista a relevância do acompanhamento da criança, tanto pelos profissionais da creche, quanto pelos da saúde. Para isso, emergiram os seguintes questionamentos: Qual a percepção materna frente 
ao crescimento e desenvolvimento da criança menor de três anos que frequenta creche? Qual a importância da creche e da Unidade de Saúde da Família (USF) para o desenvolvimento infantil? Para responder aos questionamentos, o presente estudo tem por objetivo compreender a percepção materna acerca da vigilância do desenvolvimento de crianças menores de três anos que frequentam creche.

\section{MÉTODO}

Trata-se de um estudo descritivo-exploratório com abordagem qualitativa, desenvolvido em um Centro de Referência em Educação Infantil, instituição educacional voltada para a primeira infância, do município de João Pessoa-PB que participava do Programa Saúde na Escola e estava vinculada à uma Unidade de Saúde da Família. A rede de educação infantil do município é composta por $90 \mathrm{CREI}$ vinculados à Secretaria de Educação. ${ }^{15}$

As participantes da pesquisa foram mães de crianças que contemplaram os seguintes critérios de inclusão: ser mãe de criança menor de três anos e matriculada na creche. Foram excluídas as mães com dificuldades de comunicação e aquelas menores de 18 anos.

Para a realização da coleta de dados, inicialmente foi feito contato com a Secretaria Municipal de Educação e Cultura do referido município, para autorização da pesquisa. Após isso, a pesquisadora contatou a coordenação do CREI, a fim de explicar detalhadamente a pesquisa e solicitar o seu apoio para facilitar a comunicação com as mães das crianças. A coleta de dados ocorreu logo após a autorização da coordenação do CREI e do aceite e autorização formal das mães, mediante a assinatura do Termo de Consentimento Livre e Esclarecido (TCLE).

Os dados foram coletados por meio de entrevista semiestruturada, que foi gravada em mídia digital e, posteriormente, transcrita na íntegra. $O$ encerramento da coleta de dados se deu por saturação.

Foi utilizado um roteiro semiestruturado de entrevista, contendo as seguintes questões norteadoras: i) Há o acompanhamento do crescimento e desenvolvimento do seu filho? Em qual lugar é realizado o acompanhamento do crescimento e desenvolvimento do seu filho? ii) Qual a importância do acompanhamento do crescimento e desenvolvimento? Fale um pouco sobre isso. iii) Qual é a sua percepção com relação ao desenvolvimento de seu filho que é acompanhado pela creche? iiii) Qual o papel da creche no desenvolvimento do seu filho? E o papel da unidade de saúde?

A análise dos dados seguiu os passos da análise temática propostos por Minayo, ${ }^{16}$ portanto, inicialmente, foi feita a organização dos dados abrangendo o conjunto do material coletado nas entrevistas. Em seguida, efetuamos a transcrição das entrevistas gravadas para procedermos a uma primeira organização dos relatos em determinada ordem, já iniciando uma classificação. Nessa fase, foi traçado o mapa horizontal do material. Posteriormente, à luz do referencial teórico, bem como dos objetivos propostos, realizamos a leitura exaustiva e repetida dos textos, fazendo uma relação interrogativa com eles para apreendermos as estruturas de relevância. Esse procedimento nos permitiu elaborar uma classificação por meio da leitura transversal. Em seguida, a partir das estruturas de relevância, processamos o enxugamento da classificação, reagrupando os temas mais relevantes para a análise final.

Para garantir o anonimato dos participantes, os recortes das falas foram identificados com a letra $M$ referente à mãe, seguida de uma ordenação dos números que representa a sequência das entrevistas.

Esta pesquisa foi conduzida conforme diretrizes contidas na Resolução n 466/2012 ${ }^{17}$ e vincula-se ao projeto "Vigilância do desenvolvimento infantil: caminhos e perspectivas para a Enfermagem", aprovado pelo Comitê de Ética e Pesquisa do Centro de Ciências da Saúde da Universidade Federal da Paraíba, sob o parecer nำ 2.189.497, CAAE: 02584212.3.0000.5188.

\section{RESULTADOS}

O estudo contou com a participação de nove mães, com idades entre 22 e 42 anos. No que diz respeito à escolaridade, cinco mães não concluíram o ensino médio, duas concluíram o ensino médio e duas afirmaram ter ensino superior. Em se tratando da ocupação, cinco eram do lar, três eram autônomas e uma estudante. A maioria das participantes mantinha uma relação conjugal estável ou era casada. Quanto à renda familiar mensal, sete participantes afirmaram ter renda igual ou inferior a um salário mínimo.

A partir dos relatos das mães, foi possível construir duas categorias temáticas: Fragilidade na vigilância do desenvolvimento de crianças que frequentam creche; A creche como cenário ideal para a promoção do desenvolvimento infantil.

\section{Fragilidade na vigilância do desenvolvimento de crianças que frequentam creche}

O acompanhamento da criança por profissionais de saúde, desde o seu nascimento, é fundamental para monitorar possíveis agravos, prevenir doenças e, acima de tudo, realizar a vigilância do crescimento e desenvolvimento infantil. Contudo, com os relatos analisados, percebe-se que poucas mães afirmaram levar a criança para as consultas de puericultura:

A médica acompanha ela (...) Eu fazia a puericultura dela no HU [Hospital Universitário], só que como é longe e nem toda hora eu tenho passagem, aí eu faço aqui no PSF. (M2)

Ela é acompanhada pela enfermeira lá do posto de saúde, para pesar, saber como está o desenvolvimento dela. Eu levo de seis em seis meses porque disseram que como ela é mais velha, com três anos, aí não é mais rotina levar todo mês. (M4)

Algumas mães relataram como motivo para não levarem a criança para o acompanhamento do crescimento e desenvolvimento na consulta de puericultura, a falta de médico na Unidade de Saúde da Família (USF), e para que a criança não falte a creche, 
enquanto outras apenas afirmaram que deixaram de levar a criança a USF, mas sem apontar o motivo:

No momento eu não estou levando ele para Unidade de Saúde porque está sem médico na minha área, aí deixei de ir. (M1)

Ele foi acompanhado só quando ele era bebê, até um ano, foi feito o acompanhamento por profissional de saúde. Todo mês a gente ficava fazendo, mas depois de um ano não mais. (M3)

Assim, no posto mesmo eu só fui quando ele nasceu, agora não estou indo mais porque ele fica aqui na creche durante o dia. (M6)

Quando ela era bebê, fazia todo mês a puericultura dela, até um ano e seis meses, quando ela completou dois anos eu ainda fui, mas agora não vou mais não. (M7)

Não, ele já foi para o posto, mas não tem pediatra, ai quando eu vejo qualquer coisa, qualquer problema, a gente vai na médica (...) Eu não me lembro de ter feito acompanhamento com ela [a criança] não. (M8)

Outro aspecto que emergiu nas falas das mães, é que algumas buscam os serviços da USF apenas em casos de adoecimento da criança ou para avaliar os dados antropométricos exigidos por programa assistencial, a exemplo do Programa Bolsa Família:

A última vez que eu fui, que foi para pesar do bolsa família, elas falaram que estava sem médico ainda, mandou ir terça-feira, no caso amanhã, que talvez vá ter. (M1)

Ele não vai mais para a consulta não, só vai quando precisa, quando ele está dodói, eu levo para o médico consultar entendeu? (M6)

Quando eu vejo qualquer coisa, qualquer problema, a gente vai na médica lá da clínica geral, pelo SUS. Quando ela está doente eu vou para a médica (...), não tem pediatra para estar acompanhando. (M8)

Eu só levo ela mesmo quando ela está doente, mas assim, acompanhamento fixo não, só quando era bebê. (M9)

Contudo, apesar de as participantes do estudo não possuírem o hábito de levar a criança para a puericultura, os relatos a seguir demonstram que elas reconhecem a importância desse acompanhamento rotineiro:

É necessário porque a criança pode ter três anos, nascer abaixo do peso, e indo para o acompanhamento a gente tem toda a instrução de que ela está no peso certo, de que ela tá se alimentando. (M4)

É bom para saber se a criança está evoluindo bem né?! Se tem algum problema de saúde. (M7)
É importante para saber o desenvolvimento da criança, se ela está desenvolvendo bem, se ela está crescendo bem, se ela está com o peso e altura ideal. (M9)

[é importante] para o desenvolvimento dele, tanto físico quanto emocional, era importantíssimo. Tanto para fazer as medidas, a cabeça e tudo mais, o peso dele, como estava a alimentação, então era importantíssimo para o crescimento dele, para saber se estava tudo bem com ele. (M3)

Além da unidade de saúde, a creche foi outro cenário mencionado para a realização de ações de saúde em conjunto com a USF, conforme relatos a seguir:

Teve uma reunião que veio os profissionais de saúde, vieram olhar ele, o dentista (...). (M1)

Às vezes ela chega em casa falando que teve escovação na escolinha. (M7)

Aqui [creche] eles sempre vêm, as enfermeiras da unidade vem saber das cadernetas, da vacina, tudinho... Eu acho que todo mês elas vêm, todo mês elas estão olhando porque sempre tem criança com vacina para tomar todo mês. E eu levo uma vez no mês. (M5)

\section{A creche como cenário ideal para a promoção do desenvolvimento infantil}

A inserção da criança na creche traz diversos avanços no seu desenvolvimento cognitivo, físico, emocional e comportamental, e não está marcada apenas pela necessidade materna de se inserir no mercado de trabalho, mas, as mães reconhecem que a instituição é fundamental para que a criança adquira novas habilidades e novos comportamentos sociais, reforçando a influência positiva da creche no desenvolvimento dos seus filhos:

Ela aprendeu a contar, aprendeu a pintar um pouco, aprendeu a comer sozinha. (M7)

Eu acho que a importância da creche é justamente isso, o desenvolvimento dele como pessoa também, além do nosso lar, aqui na escola ele também desenvolve o lado dele pessoal, a comunicação com os coleguinhas, com as pessoas, então eu acho de extrema importância. (M3)

Existe diferença do comportamento da criança depois que ela começou a vir para a creche sim, ela aprendeu muito mais coisas, a lidar com outras crianças também, (...) aqui ela aprende que tem que dividir os brinquedos, interage com outras crianças, desenvolveu bastante. (M4)

Ela está bem melhor, antes ela não falava muito e agora fala demais até, está aprendendo as coisas que ela chega em casa me contando, aprende muito. O desenvolvimento assim, ela não era de falar muito com ninguém e agora ela já está mais soltinha, ela come o que não comia em 
casa, a alimentação melhorou muito, come sozinha, aprendeu a fazer xixi e não usa mais fralda, tudo aqui [na creche]. (M5)

As mães percebem a creche como cenário potencializador do desenvolvimento infantil. Além disso, consideram o domicílio, juntamente com a creche, ambientes favoráveis ao desenvolvimento da criança. Entretanto, não foram todas as mães que reconheceram sua casa como um ambiente propício para o aprendizado da criança:

É melhor trazer ele para a creche né? Porque aqui ele está aprendendo também, e em casa não está. (M2)

Quando questionadas acerca da atuação dos profissionais de saúde na creche, algumas mães consideram essa ação importante para o acompanhamento da saúde da criança e reconhecem que esses setores deveriam trabalhar juntos no cuidado a criança:

Acho que não mudaria nada se os profissionais de saúde viessem até a creche para fazer o acompanhamento, acho que é do mesmo jeito. (M2)

Eu acredito que a creche e a unidade podem caminhar juntas (...) e se os profissionais [da USF] tivessem aqui [creche] seria mais fácil, com certeza. (M3)

Eu acho que eles não trabalham juntos, é a primeira vez que eu estou vendo eles aqui, e eu não vejo muita diferença não. (M4)

(...) Acharia muito bom que a creche e a unidade de saúde trabalhassem juntas, porque o médico iria estar mais perto dele né?! Sabendo se ele está bem de saúde, ou se ele não está bem (...). (M6)

\section{DISCUSSÃO}

As mães participantes do estudo reconhecem a importância da creche na promoção do desenvolvimento das crianças e da inserção dos profissionais de saúde para a vigilância do crescimento e desenvolvimento infantil nesse cenário, como institui o PSE. Porém, é diversificada a importância que dão para o acompanhamento da saúde da criança na consulta de puericultura.

A consulta de puericultura, realizada na ESF, trouxe grandes impactos nos cuidados com a criança, ${ }^{18}$ por ser esse nível de atenção à saúde um cenário propício às mudanças nas condições de saúde da população. A ESF é a responsável pela redução significativa da mortalidade infantil, bem como melhora do estado nutricional e aumento da cobertura vacinal de crianças. ${ }^{18}$

Assim, o acompanhamento na ESF e na creche é fundamental para o pleno desenvolvimento infantil, e isso já está sendo reconhecido mundialmente, quando foi declarado que o investimento na primeira infância influenciará no futuro de uma Nação, visto que crianças que alcançam seu potencial de desenvolvimento serão mais bem-sucedidas, trazendo um novo caminho para a economia mundial, ${ }^{19} \mathrm{e}$ isso inclui a inserção da criança nas instituições de educação infantil.

Apesar da relevância da promoção da VDI, os relatos das mães sugerem fragilidade no acompanhamento do crescimento e desenvolvimento na unidade de saúde, haja vista que, segundo elas, atualmente não tem sido realizado a contento, os recursos humanos no serviço são limitados e as ações de saúde são focadas no modelo biomédico de cuidado que perdura na sociedade. Esta realidade contrapõe-se ao cuidado voltado para a prevenção de doenças e promoção da saúde preconizado na APS. ${ }^{20}$ Assim, percebe-se que essas fragilidades podem influenciar na decisão materna de procurar à unidade de saúde para o seguimento do cuidado do seu filho.

Diante desse contexto, é de suma importância que as mães sejam sensibilizadas para dar continuidade às consultas na ESF, mesmo após a inserção da criança na creche e diante das fragilidades encontradas nas unidades de saúde. Isso porque, para que a criança seja assistida de forma integral, faz-se necessária a promoção da VDI por profissionais de saúde, no ambiente escolar e/ou nos serviços de saúde.

Sobre isso, uma revisão integrativa que buscou analisar o papel da enfermagem frente à promoção da saúde de crianças que frequentam creches destacou que um dos motivos referidos pelos pais para não levar suas crianças às unidades de saúde para a consulta de puericultura foi a falta de tempo. Portanto, a atuação do profissional de saúde nas creches tem potencial de sensibilizar os responsáveis das crianças, ao enfatizar a importância do seguimento infantil e a promoção de um crescimento saudável. ${ }^{12}$

Outro aspecto que chama a atenção no relato de uma mãe é que a permanência da criança no CREI em período integral foi justificativa para não realizar o acompanhamento da criança na ESF, assim como a falta do médico, demonstrando fragilidade no vínculo com a equipe de saúde e na integralidade na atenção à saúde da criança no contexto da APS. ${ }^{21}$

Corroborando esse achado, estudo que investigou a percepção de mães sobre a continuidade do cuidado à criança na APS destaca o comprometimento desse atributo, uma vez que algumas mães procuram o serviço de saúde apenas quando necessitam, ${ }^{22}$ e não rotineiramente, para o acompanhamento da criança na puericultura.

A busca para o tratamento de doenças já instaladas ainda é uma realidade na ESF, indicando que há importante demanda de assistência curativista, que está arraigada não só nos profissionais de saúde, mas também na cultura dos usuários. Apesar dos princípios da ESF ter como princípio a promoção da saúde e prevenção de agravos, além do tratamento, a população não foi preparada para tal modelo assistencial, e isso fragiliza as práticas preventivas que devem ser priorizadas no âmbito da Atenção Básica. ${ }^{23}$ 
Com isso, a saúde da criança se torna vulnerável por não haver relação terapêutica entre os atores envolvidos, e quando as intervenções são pautadas na queixa-conduta. ${ }^{21} \mathrm{Nessa}$ conjuntura, é primordial que os profissionais de saúde atentem para o cuidado integral da criança, de forma a atender às reais necessidades da população infantil e ao modelo de cuidado proposto pelo PSE. Ressalta-se ainda que as mães vincularam a consulta de acompanhamento da criança apenas a figura do médico, quando o preconizado pelo Ministério da Saúde é a execução dessa consulta, tanto pelo enfermeiro quanto pelo médico. ${ }^{11}$ Tais achados são similares a estudo no qual as mães afirmam que a puericultura deveria ser de competência do médico, especialmente o pediatra. ${ }^{24}$

Outro estudo com enfermeiros da ESF evidencia que as mães parecem não valorizar a consulta de puericultura, e centram o cuidado à criança no profissional médico, com ações predominantemente curativistas, ${ }^{25}$ corroborando os achados desse estudo, que apontam que a busca pelos serviços de saúde ocorre, em grande parte, por ocasião do adoecimento da criança.

Contrapondo a esses dados, outras mães demonstraram compreender a importância da consulta de puericultura para o acompanhamento do crescimento e desenvolvimento da criança e para a manutenção da saúde desta. Consonante a isso, estudo constatou que o conhecimento materno acerca da importância da vigilância do desenvolvimento infantil no processo saúde/doença do filho é um dos fatores que estimula as mães a buscarem a unidade de saúde para realizar o acompanhamento da criança. ${ }^{26}$

Em relação às ações de saúde realizadas pelos profissionais da ESF na creche, percebeu-se que essas acontecem de forma pontual, sem rotina programada, geralmente incluindo a análise do calendário vacinal e sua atualização, quando necessária, e ensino da escovação de dentes, sendo compatível com estudo que identificou nas ações de saúde o atendimento de situações específicas, a pedido dos profissionais da creche. ${ }^{27}$

As mães deste estudo destacaram a necessidade de articular as ações de saúde e educação, em outras palavras, implementar a intersetorialidade, que é a articulação de setores e o intercâmbio de saberes com o objetivo de integrar a conduta. Todavia, essa iniciativa ainda é pouco praticada, uma vez que a interlocução entre esses setores não é equitativa, pois há fragmentação das ações e predominância de condutas setorizadas e biomédicas. ${ }^{28}$

Destaca-se ainda baixo envolvimento dos profissionais da educação nas ações do PSE, ficando prioritariamente a cargo das equipes de saúde, com alguma participação auxiliar dos educadores em palestras e avaliações clínicas, reforçando o modelo biomédico e fragmentado da saúde. ${ }^{28,29}$

Apesar do PSE ter como princípio a integração e articulação entre as USF e os CREI de sua área de abrangência, ${ }^{10}$ faz-se necessária sensibilização e capacitação de todos os atores envolvidos acerca desse programa, para uma melhor efetivação da proposta do PSE e consequente cuidado efetivo e integral às crianças e suas famílias. Mesmo diante da relevância do exposto, estudo evidenciou baixo número de CREI que tiveram acompanhamento sistemático de profissionais da USF, mesmo diante da alta prevalência de crianças com alteração no desenvolvimento infantil, ${ }^{27}$ confirmando a necessidade de atividades conjuntas.

Dessa forma, reforça-se o importante papel do PSE para fortalecer a articulação entre a creche e a Unidade de Saúde na VDI, ao proporcionar ações para a promoção do desenvolvimento integral e o enfretamento da vulnerabilidade. Para isso, faz-se necessária a avaliação da saúde das crianças, de forma que essas possam ser encaminhadas para um acompanhamento mais específico pelos profissionais de saúde, quando necessário, bem como o incentivo para que as mães/cuidadores levem seus filhos as USF pelo menos uma vez no ano ${ }^{10}$ e sempre que necessário.

Destarte, compreende-se que as ações de saúde no âmbito da educação vão além das atividades higienistas, pois é possível efetivar e qualificar a VDI, considerando que o PSE é um programa com excelente oportunidade de promover o desenvolvimento infantil desenvolvimento saudável e prevenir agravos a este.

Outrossim, as mães reconhecem a creche como ambiente favorável para a promoção do desenvolvimento das crianças, bem como percebem mudanças positivas no comportamento dessas após sua inserção na creche, semelhante a estudo que destacou que para algumas mães a creche é o melhor ambiente para o cuidado da criança, em detrimento de cuidados alternativos de parentes e babás, e apontam a qualidade da creche como fator decisivo para tal escolha. Além disso, é o ambiente mais seguro, promove maior socialização da criança e fornece maiores estímulos ao desenvolvimento infantil. ${ }^{7}$

Os CREl têm se tornado espaço privilegiado para a promoção dos principais vínculos e estímulos necessários e para o acompanhamento do crescimento e desenvolvimento da criança. Portanto, os profissionais da creche devem estar atentos para a promoção do desenvolvimento infantil. Para isso, os profissionais da ESF devem promover ações de cuidado na creche pautadas na integralidade, entre elas, a realização de práticas educativas em saúde para os cuidadores e gestores das creches, pais e responsáveis, principalmente, das crianças identificadas com alguma alteração e/ou riscos no crescimento ${ }^{5} \mathrm{e}$ desenvolvimento.

Também é fundamental a escuta atenta às mães/cuidadores, haja vista que elas são as principais cuidadoras e, portanto, possuem maior capacidade de identificar os avanços e/ou possíveis atrasos apresentados pelo filho. ${ }^{30}$

A primeira infância tem se mostrado a janela de oportunidade que promete grandes mudanças com relação ao retorno investido em políticas e práticas que visam otimizar o desenvolvimento infantil e ajudar estas crianças a serem bem-sucedidas na vida. ${ }^{31}$ Crianças que não são estimuladas adequadamente sob o aspecto físico, mental, cognitivo e social-emocional estão propensas a consequências negativas na sua saúde, falhas na escola, comportamentos delinquentes e desemprego.

Ademais, a situação socioeconômica da família traz consigo vulnerabilidade para a criança, conferindo a ela um alto risco de atraso no desenvolvimento ou fracasso acadêmico, ${ }^{32}$ problemática 
esta que foi identificada nos dados sociodemográficos das participantes do presente estudo.

Por outro lado, a inserção precoce de crianças vulneráveis em instituições de ensino que promovam o desenvolvimento neuropsicomotor, associada aos cuidados de saúde, nutrição de alta qualidade e serviços de apoio social, promovem ganhos futuros importantes no desenvolvimento acadêmico e profissional durante a vida adulta. ${ }^{32}$

\section{CONCLUSÃO E IMPLICAÇÕES PARA A PRÁTICA}

Os resultados desse estudo apontam que as mães de crianças matriculadas em creche percebem que a vigilância do desenvolvimento infantil está fragilizada nesse espaço, uma vez que os profissionais da ESF desenvolvem apenas ações pontuais e higienistas, o que diverge do recomendado pelo PSE. Entretanto, reconhecem um salto qualitativo no desenvolvimento de seus filhos, após a inserção deles nesse espaço educativo.

Assim, para que os CREl se estabeleçam como um espaço promotor do crescimento e desenvolvimento infantil, é necessário que ofereçam ações conjuntamente com a ESF. Para isso, recomenda-se capacitação dos profissionais da saúde e da educação para a vigilância do desenvolvimento infantil e diretrizes do PSE, a fim de que ações intersetoriais sejam implementadas com vista à atenção integral à saúde das crianças, tendo em vista que inúmeras crianças com diferentes fatores de risco para o desenvolvimento permanecem a maior parte do tempo nas creches e, portanto, precisam ser acompanhadas e estimuladas.

A principal limitação foi em relação à pesquisa ter sido desenvolvida em uma única creche em que as crianças matriculadas no CREI possuíam mais de dois anos de idade, o que dificultou a inclusão de mães de crianças mais novas. Entretanto, foi possível dar visibilidade às mães sobre a sua compreensão do desenvolvimento dos seus filhos e o cuidado em saúde desses no contexto da creche e da unidade de saúde da família.

\section{CONTRIBUIÇÕES DOS AUTORES}

Desenho do estudo. Altamira Pereira da Silva Reichert. Coleta ou produção dos dados. Tayná Lima dos Santos.

Análise de dados. Altamira Pereira da Silva Reichert. Tayná Lima dos Santos. Daniele Beltrão Lucena de França. Daniele de Souza Vieira. Anniely Rodrigues Soares.

Interpretação dos resultados. Altamira Pereira da Silva Reichert. Tayná Lima dos Santos. Daniele Beltrão Lucena de França. Daniele de Souza Vieira. Anniely Rodrigues Soares.

Redação e revisão crítica do manuscrito. Altamira Pereira da Silva Reichert. Tayná Lima dos Santos. Daniele Beltrão Lucena de França. Daniele de Souza Vieira. Anniely Rodrigues Soares.

Aprovação da versão final do artigo. Altamira Pereira da Silva Reichert. Tayná Lima dos Santos. Daniele Beltrão Lucena de França. Daniele de Souza Vieira. Anniely Rodrigues Soares.

Responsabilidade por todos os aspectos do conteúdo e a integridade do artigo publicado. Altamira Pereira da Silva
Reichert. Tayná Lima dos Santos. Daniele Beltrão Lucena de França. Daniele de Souza Vieira. Anniely Rodrigues Soares.

\section{EDITOR ASSOCIADO}

\author{
Beatriz Rosana Gonçalves de Oliveira Toso
}

\section{REFERÊNCIAS}

1. Mello DF, Wernet M, Veríssimo MLR, Tonete VLP. Nursing care in early childhood: contributions from intersubjective recognition. Rev Bras Enferm. 2017;70(2):446-50. http://dx.doi.org/10.1590/0034-7167-20160319. PMid:28403283.

2. Reichert APS, Nóbrega VM, Damasceno SS, Collet N, Eickmann SH, Lima MC. Surveillance of child development: practices of nurses after training. Rev Eletr Enf. 2015;17(1):117-23. http://dx.doi.org/10.5216/ ree.v17i1.27722.

3. Almeida AC, Mendes LC, Sada IR, Ramos EG, Fonseca VM, Peixoto MVM. Use of a monitoring tool for growth and development in Brazilian children - Systematic review. Rev Paul Pediatr. 2016;34(1):122-31. http:// dx.doi.org/10.1016/j.rpped.2015.06.012. PMid:26705605.

4. Teixeira MCTV, Alckmin-Carvalho F, Emerich DR, Cevallos PV, Paula CS. Indicadores de atraso no desenvolvimento em crianças de creche advindas de famílias de baixa renda. Estud Pesqui Psicol. 2017;17(3):1042-62.

5. Torquato IMB, Dias HP, Collet N, Souza MA, Dantas MAS, Reichert APS Health surveillance in day-care centers: analysis of nutritional status in children under two years old. Rev Rene. 2018;19:e3338. http://dx.doi. org/10.15253/2175-6783.2018193338.

6. Bossi TJ, Brites S, Piccinini CA. Adjustment of Babies to Daycare: Aspects that Facilitate Adjustment or not. Paidéia (Ribeirão Preto). 2017;27(Suppl. 1):448-56. http://dx.doi.org/10.1590/1982-432727s1201710.

7. Piccini CA, Polli RG, Bortolini M, Martins GDF, Lopes RCS. Razões maternas para colocar ou não o bebê na creche. Arq Bras Psicol. 2016;3(68):59-74.

8. Corsi C, Santos MM, Marques LAP, Rocha NACF. Impact of extrinsic factors on fine motor performance of children attending day care. Rev Paul Pediatr. 2016;34(4):439-46. http://dx.doi.org/10.1016/j. rppede.2016.03.007. PMid:27094472

9. Lei $\mathrm{n}^{\circ}$ 12.796, de 4 de abril de 2013 (BR). Altera a Lei $\mathrm{n}^{\circ}$ 9.394, de 20 de dezembro de 1996, que estabelece as diretrizes e bases da educação nacional, para dispor sobre a formação dos profissionais da educação e dar outras providências. Diário Oficial da União [periódico na internet], Brasília (DF), 05 abr 2013 [citado 4 mai 2020]. Disponível em: http:// www.planalto.gov.br/ccivil_03/_Ato2011-2014/2013/Lei/L12796.htm

10. Ministério da Saúde (BR). Instrutivo Programa Saúde na Escola. $1^{\text {a }}$ ed. Brasília (DF): Ministério da Saúde; 2011 [citado 4 mai 2020]. Disponível em: http://www.saude.ba.gov.br/wp-content/uploads/2017/07/ DAB_INSTRUTIVO_PSE_2011.pdf.

11. Ministério da Saúde (BR). Saúde da criança: acompanhamento do crescimento e do desenvolvimento infantil. $1^{\text {a }}$ ed. Brasília (DF): Ministério da Saúde; 2012 [citado 4 mai 2020]. Disponível em: https:// portaldeboaspraticas.iff.fiocruz.br/wp-content/uploads/2019/07/ cadernos_atencao_basica_33.pdf

12. Alencar NMM, Pinto MB, Andrade LFD, Medeiros HA, Lima EAR, Santos NCCB. O papel da enfermagem frente à promoção da saúde de crianças que frequentam creches: uma revisão integrativa. Rev Univ Vale Rio Verde. 2017;15(2):481-91. http://dx.doi.org/10.5892/ruvrd v15i2.2925.

13. Britto PR, Lye SJ, Proulx K, Yousafzai A, Matthews SG, Vaivada $\mathrm{T}$ et al. Nurturing care: promoting early childhood development. Lancet. 2017;389(10064):91-102. http://dx.doi.org/10.1016/S01406736(16)31390-3. PMid:27717615.

14. Bezerra ICS, Santos TL, Melo AEV, França DBL, Vieira DS, Cruz TMAV et al. Análise das ações de vigilância do desenvolvimento infantil segundo cuidadores de crianças. Rev. Bras. Ciênc Saúde (Porto Alegre). 2020;24(3). http://dx.doi.org/10.22478/ufpb.2317-6032.2020v24n3.50218. 
15. João Pessoa. Centros de Referência em Educação Infantil (CREI). 2019 [citado 24 jun 2020]. Disponível em: http://www.joaopessoa.pb.gov.br/ secretarias/sedec/centros-de-referencia-em-educacao-infantil-creis/

16. Minayo MCS. O desafio do conhecimento: pesquisa qualitativa em saúde. 14 ${ }^{a}$ ed. São Paulo: HUCITEC; 2014.

17. Resolução n. 466 de 12 de dezembro de 2012 (BR). Diário Oficial da União, Brasília (DF); 2012 [citado 4 mai 2019]. Disponível em: https:// bvsms.saude.gov.br/bvs/saudelegis/cns/2013/res0466_12_12_2012. $\mathrm{html}$

18. Núcleo Ciência Pela Infância. Estudo impactos da estratégia saúde da família e desafios para o desenvolvimento infantil: estudo 5 [Internet]. São Paulo: NCPI; 2019. [citado 4 mai 2020]. Disponível em: https://ncpi.org. br/wp-content/uploads/2019/12/AF_NCPI-WP_n5_2019_online_v2.pdf

19. World Health Organization. Inheriting a sustainable world? Atlas on children's health and the environment. Geneva: WHO; 2017 [citado 10 abr 2020]. Disponível em: https://www.who.int/ceh/publications/ inheriting-a-sustainable-world/en/

20. Tavares MNM, Silva Filho JA, Silva CRL, Pinto AGA. Consulta de enfermagem em puericultura na estratégia saúde da família: revisão integrativa. Nursing (São Paulo). 2019;22(256):3144-9. http://dx.doi. org/10.36489/nursing.2019v22i256p3144-3149.

21. Diniz SGM, Damasceno SS, Coutinho SED, Toso BRGO, Collet N. Evaluating comprehensiveness in children's healthcare. Rev Gaúcha Enferm. 2016;37(4):e57067. http://dx.doi.org/10.1590/19831447.2016.04.57067. PMid:27992031.

22. Nóbrega VM, Reichert APS, Viera CS, Collet N. Longitudinality and continuity of care for children and adolescents with chronic diseases. Esc Anna Nery. 2015;19(4):656-63. http://dx.doi.org/10.5935/14148145.20150088.

23. Brito GEG, Mendes ACG, Santos Neto PM. O trabalho na estratégia saúde da família e a persistência das práticas curativistas. Trab Educ Saúde. 2018;16(3):975-95. http://dx.doi.org/10.1590/1981-7746sol00164.

24. Malaquias TSM, Gaíva MAM, Higarashi IH. Perceptions of the family members of children regarding well-child check-ups in the family healthcare strategy. Rev Gaúcha Enferm. 2015;36(1):62-8. http://dx.doi. org/10.1590/1983-1447.2015.01.46907. PMid:26098804.

25. Vieira DS, Dias TKC, Pedrosa RKB, Vaz EMC, Collet N, Reichert APS Work process of nurses in child development surveillance. Rev Min Enferm. 2019;23:e-1242.http://dx.doi.org/10.5935/1415-2762.20190090.

26. Rodrigues DA, Sousa MD, Silva FJS, Carvalho DPSRP, Bezerra STF, Gomes JGN. Avaliação da adesão às consultas de crescimento e desenvolvimento infantil. Rev enferm UFPE on line. 2019;13(4):1023-9. https://doi.org/10.5205/1981-8963-v13i04a238262p1023-1029-2019.

27. Silva ACD, Engstron EM, Miranda CT. Fatores associados ao desenvolvimento neuropsicomotor em crianças de 6-18 meses de vida inseridas em creches públicas do Município de João Pessoa, Paraíba, Brasil. Cad Saude Publica. 2015;31(9):1881-93. http://dx.doi. org/10.1590/0102-311X00104814. PMid:26578013.

28. Brito GEG, Mendes ACG, Santos Neto PM. O trabalho na estratégia saúde da família e a persistência das práticas curativistas. Trab Educ Saúde. 2018;16(3):975-95. http://dx.doi.org/10.1590/1981-7746sol00164.

29. Silva-Sobrinho RA, Pereira BSA, Trevisan CL, Martins FJ, Almeida ML, Mansour NR et al. Percepção dos profissionais da educação $e$ saúde sobre o programa saúde na escola. Revista Pesquisa Qualitativa [Internet]. 2017; [citado 10 jan 2021];5(7):93-108. Disponível em: https:// editora.sepq.org.br/rpq/article/view/77/66

30. Trezzi C, Rosa GRA. Os ambientes educativos na creche e sua influência no desenvolvimento da criança. Revista Ambiente Educação. 2020;13(1):17690. http://dx.doi.org/10.26843/ae19828632v13n12020p176a190

31. Guhn M, Janus M, Enns J, Brownell M, Forer B, Duku E et al. Examining the social determinants of children's developmental health: protocol for building a pan-Canadian population-based monitoring system for early childhood development. BMJ Open. 2016;6(4):e012020. http://dx.doi. org/10.1136/bmjopen-2016-012020. PMid:27130168.

32. Campbell FA, Pan Y, Burchinal M. Sustaining gains from early childhood intervention: the abecedarian program. In: Reynolds A, Temple J. Sustaining early childhood learning gains: Program, school, and family influences. New York: Cambridge University Press; 2019. p. 268-86. http://dx.doi.org/10.1017/9781108349352.013 